\title{
Evaluation of Different Maize Hybrids for Morpho-physiological, Biochemical and Yield and Yield Components
}

\author{
K. N. Pawar* and A. L. Goudar \\ University of Agricultural Sciences Dharwad, Karnataka, India \\ *Corresponding author
}

\begin{tabular}{|c|}
\hline Keywords \\
\hline $\begin{array}{l}\text { Maize genotypes, } \\
\text { Photosynthetic rate, } \\
\text { Chlorophyll } \\
\text { content, Yield traits }\end{array}$ \\
\hline Article Info \\
\hline $\begin{array}{l}\text { Accepted: } \\
16 \text { November } 2020 \\
\text { Available Online: } \\
10 \text { December } 2020\end{array}$ \\
\hline
\end{tabular}

\section{A B S T R A C T}

A field experiment was conducted during Kharif 2015-16 at Main Agricultural Research Station, University of Agricultural Sciences, Dharwad to find out the physiological basis of yield variation of different maize hybrids. The experiment consisted of twenty maize hybrids replicated thrice in randomized block design. Observations recorded were morphophysiological, biochemical, and yield and yield attributes. The hybrids DMH-01, DMH-13 and GPMH-1101 exhibited superiority over rest of the hybrids with respect to higher morphological (plant height, more number of leaves and total dry matter) and physiological parameters (SPAD, Chlorophyll, total sugar and starch content).Significantly higher yield and yield parameters such as cob length, cob girth, number of seeds per cob, number of seed rows per cob, 100-kernel weight and harvest index were recorded by DMH-01, DMH-13 and GPMH-1101 which increased the yield of maize hybrids. Significant improvement in overall growth of the crop was due to its increased photosynthetic efficiency which resulted in greater availability of photosynthates, metabolites and increased dry matter accumulation for growth and development of reproductive structures which has contributed for higher productivity.

\section{Introduction}

Maize (Zea mays L.) is the world's leading crop and is widely cultivated as cereal grain that was domesticated in Central America. It is one of the most versatile emerging crops having wider adaptability. Globally, maize is known as queen of cereals because of its highest genetic yield potential. Maize is the only food cereal crop that can be grown in diverse seasons, ecologies and uses. Beside this maize have many types like normal yellow/ white grain, sweet corn, baby corn, popcorn, waxy corn, high amylase corn, high oil corn, quality protein maize, etc. Apart from this, maize is an important industrial raw material and provides large opportunity for value addition.

In India, maize is the third important food crop after rice and wheat.. Maize is primarily used for feed $(60 \%)$ followed by human food $(24 \%)$, industrial (starch) products (14\%) beverages and seed (1\% each). Thus, maize has attained an important position as industrial crop because $75 \%$ of its produced is 
used in starch and feed industries. In India, maize is predominantly cultivated as rainfed crop but due to focused research on single cross hybrids. The projected growth rates suggest that maize demand is expected to increase from current level of 22 to $45 \mathrm{M}$. tonnes by 2030. Maize is truly a forward looking crop especially in the context of climate change. It is an annual, short day, cross pollinated, photosynthetically more active $\left(\mathrm{C}_{4}\right)$ and which is grown extensively in temperate, subtropical and tropical regions of the world.

In addition to staple crop for human being and quality feed for animals, maize serves as a basic raw material for production of starch, oil, protein, alcohol beverages, food sweeteners and more recently as a potential bio-fuel crop in India. Maize occupies important place as food (25\%), animal feed $(12 \%)$ poultry feed $(49 \%)$, industrial products mainly starch (12\%) and one per cent each in brewery and seed (Dass et al., 2008). Maize has attained a commercial crop status due to easiness in cultivation, tolerance towards pest and diseases, high yield and better market price. It comes up well under a wide range of soil and climatic conditions. There is great variations occur in the yields of maize and these large differences in yield are not only be accounted by climate or soil variability alone, since areas with the same climate and rainfall pattern show markedly different average yields. The present study was conducted to evaluate the hybrid performance for the morpho-physiological, biochemical quality aspects such as starch and total sugars and yield and its components

\section{Materials and Methods}

The present study consisted of twenty maize hybrids viz., Arjun, GH-0727, GPMH-1101, GPMH-1111, GH-1018, GH-1043， GH110145, GH-110204, DMH-01, DMH-03,
DMH10, DMH-13, NAH-1137, NAH-2049, MAH-957, МАH-974 and were evaluated in randomized complete block design with three replications at Main Agricultural Research Station, University of Agricultural Sciences, Dharwad during kharif 2015-16. The quality attributes such as starch content, total sugar content and morpho-physiological parameters of these genotypes and their yield attributes were studied. The plant height was measured from base of the plant to tip of the leaf. Manual counting of leaf and assessment of dry matter was recorded during reproductive stage. Leaf area of all green leaves from five tagged plant was measured by adopting Stickler's linear measurement method and total chlorophyll content was determined by following DMSO method of Hiscox and Israeltam, 1979. The absorbance of the extract was measured at 645, 652 and $663 \mathrm{~nm}$ in a UV- VIS spectrophotometer (Elico, SL -159). The amount of starch and soluble sugars present in the maize kernels was estimated by Anthrone method.

\section{Results and Discussion}

Various morpho-physiological characters like plant height, number of leaves, leaf area and total dry matter production at reproductive stages were studied. Though the plant height is genetically controlled character, it is being influenced by environmental conditions and management practices. The results of the present investigation revealed the significant differences in plant height of maize at reproductive stages. Of the 20 genotypes tested, DMH-01 was recorded significantly higher plant height at harvest $(193.43 \mathrm{~cm})$ as compared to other genotypes and the lowest plant height of $154.57 \mathrm{~cm}$ was noticed in DMH-08 (Table 1). The hybrids GPMH, DMH-03, DMH-10, DMH-13, NAH-13, NAH-1137, NAH-2049, MAH-957 and MAH974 were found to be on par with each other $(192.79,189.30,193.66,193.66$, 
191.16, 190.32, 191.32, 191.50 and 188.77 respectively). It was found that plant height was positively correlated with grain yield per unit area. (Saleem et al., 2007) reported that, plant height showed positive and direct effect on grain yield. Increase in grain yield with an increase in plant height during later stages could be attributed to the better utilization of stem reserves and also to the canopy architecture i.e., increase in plant height alters the spatial arrangement of leaves leading to higher leaf area and better light distribution in the canopy The leaf number per plant differed significantly among the hybrids. Among the hybrids DMH-01 and DMH-13 recorded significantly higher leaf number per plant (13.24 and 13.24 respectively) compared to other hybrids. Hybrid Arjun recorded significantly lower leaf number (11.36). Such a variation in number of leaves was also reported by (Modarres et al., 1997) where they also observed the effect of genotype and ear and the interaction of the two were significant for leaf number results in greater yield. The data on leaf area was recorded at reproductive stages of maize genotypes are presented in table 1 .

Leaf area differed significantly among the genotypes. In general, leaf area increased up to 60 days after sowing. DMH-01 recorded significantly higher leaf area $\left(108.2 \mathrm{dm}^{2}\right.$ plant $\left.^{-1}\right)$ followed by DHM-13 (100.5 dm plant $\left.^{-1}\right)$. The lowest leaf area was recorded in hybrid DMH-04 (94.2 dm plant $\left.^{-1}\right)$. Therefore formed useful indirect indicator for yield improvement in maize crop. These results are in conformity with the experiments conducted by (Hader, 2006) and (Banziger and Araus, 2007). The data on total dry weight presented in Table 1. In general, total dry weight increased with the age of the crop and maximum at harvest. At reproductive stage, there was significant differences were recorded among the hybrids, although numerically the maximum total dry matter accumulation (181.29 $\mathrm{g} \mathrm{plant}^{-1}$ ) was recorded with genotype DMH-01 followed by MAH957 and DMH-13 (177.95 and $176.85 \mathrm{~g}$ plant $^{-1}$ respectively), while least (163.45 g plant $^{-1}$ ) total dry matter accumulation was recorded with DMH-04. The hybrids NAH1137 and NAH-2049 were found to be on par with each other.

Long duration hybrids recorded more total dry weights compared to short duration hybrids at all the growth stages. This might be due to genotypic difference in the translocation of source-sink ratio. Further resulting in better uptake of nutrients, increased vegetative growth, PAR and RUE throughout the growth period leads to increased accumulation of dry matter in the different parts of the plant. These results are in conformity with the findings of (Manish kumar 1998). It was also noticed that, the higher dry matter in reproductive parts increased with an increase in total dry weight.

Crop yield is mainly dependent on the interplay of various physiological and biochemical functions of the plant in addition to the impact of environment. Chlorophyll is a natural pigment that traps light which is essential for the process of photosynthesis. A close relationship between leaf chlorophyll content and photosynthetic rate was observed. The data on SPAD values which are influenced by different maize genotypes recorded at reproductive stages presented in table 2. In general, SPAD values increased with the age of the crop from 45 to 60 days after sowing. At reproductive stage, the highest SPAD value (47.61) was recorded with DMH-01 which was superior over all maize genotypes except DMH-04, DMH-08 and DMH-17 and DMH-06 which were statistically significant with DMH-01.

However, lower SPAD value (34.09) was recorded with DMH-04 genotype. such results 
were also noticed by (Peng et al., 1996), (Balasubramanian et al., 1999) and (Pellizzaro et al., 1998). In general, total chlorophyll content was increased with age of the crop from 30 DAS to 60 DAS and thereafter decreases till harvest. At reproductive stage, DMH-01 recorded significantly high chlorophyll content (2.81 $\mathrm{mg} \mathrm{g}^{-1}$ fr. wt.) followed by DMH-13 $(2.34 \mathrm{mg}$ $\mathrm{g}^{-1}$ fr. wt.), GPMH-1101 (2.08 $\mathrm{mg} \mathrm{g}^{-1}$ fr. wt.) and MAH-957 (2.03 $\mathrm{mg} \mathrm{g}^{-1}$ fr. wt.). Results of present study indicated that total chlorophyll was maximum at silking period and among the maize genotypes, DMH-01 recorded higher total chlorophyll content during cob initiation period and the lowest chlorophyll content was recorded in low yielding DMH-
04 genotype. Similar results were reported by (Yunfeng et al., 2014). The data on starch content of different maize genotypes presented in (Table 2).

After the harvest of the crop starch content was measured in kernels, maximum starch content $\left(61.7 \mathrm{mg} \mathrm{g}^{-1}\right)$ was recorded with DMH-01, which differ significantly superior with all other genotypes followed by $\mathrm{DMH}-$ 13 (59.8mg g ${ }^{-1}$ ), GPMH-1101 (58.6 $\mathrm{mg} \mathrm{g}^{-1}$ ) and MAH-957 (56.9 $\left.\mathrm{mg} \mathrm{g}^{-1}\right)$. Whereas, least starch content $\left(32.5 \mathrm{mg} \mathrm{g}^{-1}\right)$ was recorded with DMH-04. The data on starch content was in agreement with the study by (Yunfeng et al., 2014).

Table.1 Genotypic differences in plant height, number of leaves, leaf area and total dry matter at reproductive stages of maize

\begin{tabular}{|c|c|c|c|c|}
\hline Genotypes & $\begin{array}{l}\text { Plant height } \\
\text { (cm) }\end{array}$ & $\begin{array}{l}\text { Number of } \\
\text { Leaves }\end{array}$ & $\begin{array}{l}\text { Leaf area }\left(\mathrm{dm}^{2}\right. \\
\left.\text { plant }^{-1}\right)\end{array}$ & $\begin{array}{l}\text { Total dry of leaves } \\
\text { matter }\left(\mathrm{g} \mathrm{plant}^{-1}\right)\end{array}$ \\
\hline Arjun & $178.99 \mathrm{a}-\mathrm{c}$ & $11.36 f-g$ & $90.5 b-d$ & 167.35 \\
\hline GH-0727 & $184.04 \mathrm{ab}$ & $11.69 \mathrm{~d}-\mathrm{f}$ & $82.1 \mathrm{c}-\mathrm{f}$ & 165.82 \\
\hline GPMH-1101 & $192.79 a$ & $13.13 \mathrm{ab}$ & $94.2 b c$ & 171.72 \\
\hline GPMH-1111 & $185.16 \mathrm{ab}$ & $12.02 \mathrm{c}-\mathrm{e}$ & $91.8 b-d$ & 169.18 \\
\hline GH-1018 & $187.86 \mathrm{a}$ & $12.24 \mathrm{~cd}$ & $90.7 b-d$ & 170.25 \\
\hline GH-1043 & $171.56 \mathrm{a}-\mathrm{c}$ & $10.80 \mathrm{gh}$ & $84.1 \mathrm{c}-\mathrm{e}$ & 168.78 \\
\hline GH-110145 & $175.61 \mathrm{a}-\mathrm{c}$ & $10.91 \mathrm{gh}$ & $87.1 \mathrm{~b}-\mathrm{e}$ & 166.40 \\
\hline GH-110204 & $178.03 \mathrm{a}-\mathrm{c}$ & $11.13 \mathrm{f}-\mathrm{h}$ & $81.7 \mathrm{c}-\mathrm{f}$ & 169.54 \\
\hline DMH-01 & $193.43 a$ & $13.24 \mathrm{a}$ & $108.2 \mathrm{a}$ & 181.29 \\
\hline DMH-03 & $189.30 \mathrm{a}$ & $12.35 \mathrm{~cd}$ & $79.5 \mathrm{~d}-\mathrm{f}$ & 173.15 \\
\hline DMH-10 & $189.96 a$ & $12.46 b c$ & $86.0 \mathrm{c}-\mathrm{e}$ & 173.59 \\
\hline DMH-13 & $193.66 \mathrm{a}$ & $13.24 \mathrm{a}$ & $100.5 \mathrm{ab}$ & 176.75 \\
\hline NAH-1137 & $191.16 \mathrm{a}$ & $12.58 \mathrm{a}-\mathrm{c}$ & $86.6 \mathrm{~b}-\mathrm{e}$ & 175.17 \\
\hline NAH-2049 & $190.32 \mathrm{a}$ & $12.58 \mathrm{a}-\mathrm{c}$ & $81.4 \mathrm{c}-\mathrm{f}$ & 174.88 \\
\hline МАН-957 & $191.50 \mathrm{a}$ & 13. $13 \mathrm{ab}$ & $91.0 \mathrm{~b}-\mathrm{d}$ & 177.95 \\
\hline МАН-974 & $188.77 \mathrm{a}$ & $12.35 \mathrm{~cd}$ & 74.9ef & 168.62 \\
\hline DMH-04 & $151.49 \mathrm{c}$ & $9.91 \mathrm{i}$ & $68.4 f$ & 169.19 \\
\hline DMH-06 & $167.66 \mathrm{a}-\mathrm{c}$ & 10.58hi & 78.0d-f & 163.45 \\
\hline DMH-08 & $156.02 b c$ & $10.47 \mathrm{hi}$ & $73.5 \mathrm{ef}$ & 170.56 \\
\hline DMH-17 & $164.00 \mathrm{a}-\mathrm{c}$ & 10.58hi & 75.0ef & 168.92 \\
\hline $\begin{array}{c}\text { S.Em+ } \\
\text { LSD }(5 \%)\end{array}$ & $\begin{array}{l}3.14 \\
8.98\end{array}$ & $\begin{array}{l}0.23 \\
0.67\end{array}$ & $\begin{array}{c}5.1 \\
14.6\end{array}$ & $\begin{array}{l}1.15 \\
\text { NS }\end{array}$ \\
\hline
\end{tabular}

Note: DMRT-values in the column followed by the same letter do not differ significantly

DAS - Days after sowing NS: Non significant 
Table. 2 Genotypic differences in SPAD values, chlorophyll content, starch content $\left(\mathrm{mg} \mathrm{g}^{-1}\right)$ and total sugar content at reproductive stages of maize

\begin{tabular}{|c|c|c|c|c|c|c|}
\hline SL No. & Genotypes & $\begin{array}{l}\text { SPAD } \\
\text { values }\end{array}$ & $\begin{array}{c}\text { Chlorophyll } \\
\text { content } \\
\text { (mg g } \text { gr.Wt.) }^{-1} \text { fr. }\end{array}$ & $\begin{array}{l}\text { Starch } \\
\text { content } \\
\left(\mathbf{m g ~ g}^{-1}\right)\end{array}$ & $\begin{array}{c}\text { Total sugar } \\
\text { content } \\
\left(\mathrm{mg} \mathrm{g}^{-1}\right)\end{array}$ & $\begin{array}{l}\text { Photosynthetic rate } \\
\left(\mu \mathrm{mol} \mathrm{CO} \mathrm{m}^{-2} \mathrm{~s}^{-1}\right)\end{array}$ \\
\hline 1. & Arjun & $41.19 a-d$ & $1.76 f g$ & $41.2 \mathrm{e}$ & $0.77 \mathrm{~g}$ & $21.49 \mathrm{f}-\mathrm{h}$ \\
\hline 2. & GH-0727 & $41.69 \mathrm{a}-\mathrm{d}$ & $1.82 \mathrm{e}-\mathrm{g}$ & $45.7 \mathrm{~cd}$ & $0.79 \mathrm{~g}$ & $21.58 \mathrm{f}-\mathrm{h}$ \\
\hline 3. & GPMH-1101 & $46.61 \mathrm{ab}$ & $2.08 c$ & $58.6 \mathrm{ab}$ & $1.19 \mathrm{c}$ & $28.42 \mathrm{ab}$ \\
\hline 4. & GPMH-1111 & $42.79 a-c$ & $1.90 \mathrm{c}-\mathrm{f}$ & $47.3 \mathrm{~cd}$ & $0.80 \mathrm{~g}$ & $22.23 \mathrm{e}-\mathrm{h}$ \\
\hline 5. & GH-1018 & $42.81 \mathrm{a}-\mathrm{c}$ & $1.91 \mathrm{c}-\mathrm{f}$ & $47.8 \mathrm{~cd}$ & $0.83 f g$ & $23.19 d-g$ \\
\hline 6. & GH-1043 & $39.49 \mathrm{a}-\mathrm{d}$ & $1.89 \mathrm{c}-\mathrm{f}$ & $36.5 f$ & $0.52 \mathrm{hi}$ & $18.01 \mathrm{i}$ \\
\hline 7. & GH-110145 & $39.76 a-d$ & $1.82 \mathrm{e}-\mathrm{g}$ & $35.2 f$ & $0.57 \mathrm{hi}$ & $20.07 \mathrm{~h}$ \\
\hline 8. & GH-110204 & $40.05 a-d$ & $1.77 \mathrm{fg}$ & $35.1 \mathrm{f}$ & $0.60 \mathrm{~h}$ & $21.12 \mathrm{gh}$ \\
\hline 9. & DMH-01 & $47.61 \mathrm{a}$ & $2.81 \mathrm{a}$ & $61.7 \mathrm{a}$ & $2.92 \mathrm{a}$ & $29.57 \mathrm{a}$ \\
\hline 10. & DMH-03 & $45.06 \mathrm{a}-\mathrm{c}$ & $1.89 \mathrm{c}-\mathrm{f}$ & $45.2 \mathrm{c}-\mathrm{e}$ & $0.92 \mathrm{ef}$ & 23.96de \\
\hline 11. & DMH-10 & $45.81 \mathrm{ab}$ & $1.98 \mathrm{c}-\mathrm{e}$ & $49.1 c$ & $0.95 \mathrm{e}$ & $23.37 \mathrm{~d}-\mathrm{g}$ \\
\hline 12. & DMH-13 & $47.32 \mathrm{a}$ & $2.34 \mathrm{~b}$ & $59.8 \mathrm{ab}$ & $2.56 \mathrm{~b}$ & $28.76 \mathrm{a}$ \\
\hline 13. & NAH-1137 & $45.34 \mathrm{ab}$ & $1.88 \mathrm{~d}-\mathrm{f}$ & $56.2 \mathrm{~b}$ & $1.08 \mathrm{~cd}$ & $26.52 b c$ \\
\hline 14. & NAH-2049 & $44.34 a-c$ & $1.91 \mathrm{c}-\mathrm{f}$ & $45.9 \mathrm{~cd}$ & $0.98 \mathrm{de}$ & $24.56 \mathrm{~cd}$ \\
\hline 15. & МАH-957 & $46.12 \mathrm{ab}$ & $2.03 \mathrm{~cd}$ & $56.9 \mathrm{~b}$ & $1.10 \mathrm{c}$ & $28.31 \mathrm{ab}$ \\
\hline 16. & МАН-974 & $43.21 \mathrm{a}-\mathrm{c}$ & $1.88 \mathrm{~d}-\mathrm{f}$ & $43.2 \mathrm{de}$ & $0.88 \mathrm{e}-\mathrm{g}$ & $23.65 d-f$ \\
\hline 17. & DMH-04 & $34.09 \mathrm{~d}$ & $1.38 \mathrm{~h}$ & $32.5 \mathrm{f}$ & $0.28 \mathrm{j}$ & $10.97 \mathrm{k}$ \\
\hline 18. & DMH-06 & $38.38 b-d$ & $1.47 \mathrm{~h}$ & $33.2 \mathrm{f}$ & $0.47 i$ & $16.91 \mathrm{i}$ \\
\hline 19. & DMH-08 & $36.37 \mathrm{~cd}$ & $1.45 \mathrm{~h}$ & $34.3 \mathrm{f}$ & $0.35 j$ & $14.69 \mathrm{j}$ \\
\hline \multirow[t]{2}{*}{20.} & DMH-17 & $38.38 \mathrm{~b}-\mathrm{d}$ & $1.65 \mathrm{~g}$ & $35.1 \mathrm{f}$ & $0.46 \mathrm{i}$ & 16.00ij \\
\hline & $\begin{array}{c}\text { S.Em+ } \\
\text { LSD (5\%) }\end{array}$ & $\begin{array}{l}0.39 \\
1.11\end{array}$ & $\begin{array}{l}0.06 \\
0.17\end{array}$ & $\begin{array}{l}1.4 \\
4.1\end{array}$ & $\begin{array}{l}0.04 \\
0.11\end{array}$ & $\begin{array}{l}0.70 \\
2.01\end{array}$ \\
\hline
\end{tabular}

Note: DMRT-values in the column followed by the same letter do not differ significantly

DAS - Days after sowing

Table.3 Genotypic differences of cob weight $(\mathrm{g})$, Cob girth $(\mathrm{cm})$, cob length $(\mathrm{cm})$, No. of seed rows $\operatorname{cob}^{-1}$ and No. of seeds $\mathrm{cob}^{-1}$

\begin{tabular}{|c|c|c|c|c|c|c|}
\hline SL No. & Genotype & $\begin{array}{c}\text { Cob } \\
\text { Weight (g) }\end{array}$ & $\begin{array}{l}\text { Cob girth } \\
\text { (cm) }\end{array}$ & $\begin{array}{l}\text { Cob length } \\
\text { (cm) }\end{array}$ & $\begin{array}{c}\text { No. of } \\
\text { seed rows } \text { cob }^{-1}\end{array}$ & $\begin{array}{c}\text { No. of } \\
\text { seeds cob-1 }\end{array}$ \\
\hline 1. & Arjun & $161.33 \mathrm{e}-\mathrm{h}$ & $15.75 \mathrm{ab}$ & $14.61 \mathrm{~d}-\mathrm{g}$ & $14.27 b-f$ & 330.08 g-i \\
\hline 2. & GH-0727 & $167.33 d-g$ & $15.99 \mathrm{ab}$ & $14.77 d-g$ & $14.27 b-f$ & $349.84 f-i$ \\
\hline 3. & GPMH-1101 & $199.00 \mathrm{bc}$ & 17.06ab & $17.47 a-c$ & $16.13 a-c$ & $428.95 \mathrm{~b}-\mathrm{e}$ \\
\hline 4. & GPMH-1111 & $170.67 \mathrm{c}-\mathrm{g}$ & $16.15 \mathrm{ab}$ & $15.15 c-f$ & $14.40 \mathrm{~b}-\mathrm{f}$ & $355.34 \mathrm{f}-\mathrm{i}$ \\
\hline 5. & GH-1018 & $174.33 \mathrm{c}-\mathrm{g}$ & $16.19 \mathrm{ab}$ & $15.24 c-f$ & $14.40 \mathrm{~b}-\mathrm{f}$ & $399.55 c-f$ \\
\hline 6. & GH-1043 & $147.33 \mathrm{~g}-\mathrm{i}$ & $15.41 \mathrm{ab}$ & $13.61 \mathrm{f}-\mathrm{h}$ & $13.60 \mathrm{c}-\mathrm{f}$ & $382.44 \mathrm{e}-\mathrm{h}$ \\
\hline 7. & GH-110145 & $151.00 f-i$ & $15.65 \mathrm{ab}$ & $14.05 \mathrm{e}-\mathrm{g}$ & $13.60 c-f$ & $390.50 \mathrm{~d}-\mathrm{g}$ \\
\hline 8. & GH-110204 & $157.33 f-\mathrm{i}$ & $15.71 \mathrm{ab}$ & $14.31 \mathrm{e}-\mathrm{g}$ & $13.73 b-f$ & $517.00 \mathrm{a}$ \\
\hline 9. & DMH-01 & $234.00 \mathrm{a}$ & $17.71 \mathrm{a}$ & $18.12 \mathrm{a}$ & $17.47 \mathrm{a}$ & $520.77 \mathrm{a}$ \\
\hline 10. & DMH-03 & $179.00 \mathrm{c}-\mathrm{f}$ & $16.49 \mathrm{ab}$ & $15.57 \mathrm{~b}-\mathrm{f}$ & $14.67 \mathrm{~b}-\mathrm{f}$ & $437.02 \mathrm{~b}-\mathrm{e}$ \\
\hline 11. & DMH-10 & $183.67 \mathrm{c}-\mathrm{e}$ & $16.57 \mathrm{ab}$ & $15.77 a-f$ & $14.93 b-f$ & $425.60 \mathrm{~b}-\mathrm{e}$ \\
\hline
\end{tabular}




\begin{tabular}{|c|c|c|c|c|c|c|}
\hline $\mathbf{1 2 .}$ & DMH-13 & $220.00 \mathrm{ab}$ & $17.14 \mathrm{ab}$ & $17.87 \mathrm{ab}$ & $16.27 \mathrm{ab}$ & $432.91 \mathrm{~b}-\mathrm{e}$ \\
\hline $\mathbf{1 3 .}$ & NAH-1137 & $191.33 \mathrm{c} d$ & $16.84 \mathrm{ab}$ & $16.59 \mathrm{a}-\mathrm{e}$ & $15.73 \mathrm{a}-\mathrm{d}$ & $458.40 \mathrm{a}-\mathrm{d}$ \\
\hline $\mathbf{1 4 .}$ & NAH-2049 & $185.67 \mathrm{c}-\mathrm{e}$ & $16.69 \mathrm{ab}$ & $16.27 \mathrm{a}-\mathrm{e}$ & $15.33 \mathrm{a}-\mathrm{e}$ & $470.92 \mathrm{ab}$ \\
\hline $\mathbf{1 5 .}$ & MAH-957 & $195.67 \mathrm{~b}-\mathrm{d}$ & $16.90 \mathrm{ab}$ & $17.07 \mathrm{a}-\mathrm{d}$ & $15.87 \mathrm{a}-\mathrm{c}$ & $482.41 \mathrm{ab}$ \\
\hline $\mathbf{1 6 .}$ & MAH-974 & $175.67 \mathrm{c}-\mathrm{g}$ & $16.31 \mathrm{ab}$ & $15.39 \mathrm{~b}-\mathrm{f}$ & $14.53 \mathrm{~b}-\mathrm{f}$ & $461.79 \mathrm{a}-\mathrm{c}$ \\
\hline $\mathbf{1 7 .}$ & DMH-04 & $119.00 \mathrm{j}$ & $14.34 \mathrm{~b}$ & $11.54 \mathrm{~h}$ & $12.67 \mathrm{f}$ & $327.37 \mathrm{~g}-\mathrm{i}$ \\
\hline $\mathbf{1 8 .}$ & DMH-06 & $137.00 \mathrm{~h}-\mathrm{j}$ & $15.34 \mathrm{ab}$ & $13.38 \mathrm{f}-\mathrm{h}$ & $13.60 \mathrm{c}-\mathrm{f}$ & $431.82 \mathrm{~b}-\mathrm{e}$ \\
\hline $\mathbf{1 9 .}$ & DMH-08 & $132.33 \mathrm{ij}$ & $14.64 \mathrm{ab}$ & $12.25 \mathrm{gh}$ & $13.07 \mathrm{ef}$ & $314.19 \mathrm{hi}$ \\
\hline $\mathbf{2 0 .}$ & DMH-17 & $134.00 \mathrm{~h}-\mathrm{j}$ & $15.06 \mathrm{ab}$ & $13.21 \mathrm{f}-\mathrm{h}$ & $13.20 \mathrm{~d}-\mathrm{f}$ & $295.16 \mathrm{i}$ \\
\hline & Mean & $\mathbf{1 7 0 . 7 8}$ & $\mathbf{1 6 . 1 0}$ & $\mathbf{1 5 . 1 1}$ & $\mathbf{1 4 . 5 9}$ & $\mathbf{4 1 0 . 6 0}$ \\
\hline & S.Em+ & 6.655 & 0.17 & 14.99 & 0.25 & 33.01 \\
\hline & LSD(5\%) & 19.052 & 0.50 & 0.45 & 0.73 & 94.51 \\
\hline
\end{tabular}

Note: DMRT-values in the column followed by the same letter do not differ significantly

DAS - Days after sowing

Table.4 Genotypic differences of test weight, yield plant ${ }^{-1}$, yield ( $\left.\mathrm{q} \mathrm{ha}^{-1}\right)$, shelling (\%), harvest index (\%)

\begin{tabular}{|c|c|c|c|c|c|c|}
\hline SL No. & Genotype & $\begin{array}{c}\text { Test } \\
\text { weight }(g)\end{array}$ & $\begin{array}{l}\text { Yield plant } \\
\text { (g) }\end{array}$ & $\begin{array}{c}\text { Yield } \\
\left(\mathbf{q} \text { ha }^{-1}\right)\end{array}$ & $\begin{array}{c}\text { Shelling } \\
(\%)\end{array}$ & $\begin{array}{c}\text { Harvest } \\
\text { index }(\%)\end{array}$ \\
\hline 1. & Arjun & $43.3 \mathrm{a}$ & $138.67 f-j$ & $77.65 f-j$ & 86.00 & $49.76 a-c$ \\
\hline 2. & GH-0727 & $40.9 \mathrm{a}$ & $141.33 f-j$ & $79.15 f-j$ & 84.53 & $46.30 \mathrm{a}-\mathrm{c}$ \\
\hline 3. & GPMH-1101 & $41.5 \mathrm{a}$ & $177.67 \mathrm{a}-\mathrm{c}$ & $99.49 a-c$ & 89.25 & $52.70 \mathrm{ab}$ \\
\hline 4. & GPMH-1111 & $41.7 \mathrm{a}$ & $143.67 e-j$ & $80.45 e-j$ & 84.18 & $48.67 a-c$ \\
\hline 5. & GH-1018 & $38.3 \mathrm{ab}$ & $152.33 d-i$ & $85.31 d-i$ & 87.40 & $50.83 a b$ \\
\hline 6. & GH-1043 & $33.8 \mathrm{a}-\mathrm{d}$ & $128.33 \mathrm{i}-\mathrm{k}$ & $71.87 \mathrm{i}-\mathrm{k}$ & 87.24 & $47.82 \mathrm{a}-\mathrm{c}$ \\
\hline 7. & GH-110145 & $33.5 a-d$ & $130.33 \mathrm{~h}-\mathrm{k}$ & 72.99h- k & 86.38 & $46.50 \mathrm{a}-\mathrm{c}$ \\
\hline 8. & GH-110204 & $26.3 d$ & $135.00 \mathrm{~g}-\mathrm{k}$ & $75.60 \mathrm{~g}-\mathrm{k}$ & 85.77 & $49.04 a-c$ \\
\hline 9. & DMH-01 & $37.2 \mathrm{a}-\mathrm{c}$ & $192.33 \mathrm{a}$ & $107.71 \mathrm{a}$ & 82.21 & $52.39 \mathrm{ab}$ \\
\hline 10. & DMH-03 & $35.8 \mathrm{a}-\mathrm{c}$ & $155.33 \mathrm{~b}-\mathrm{g}$ & $86.99 \mathrm{~b}-\mathrm{g}$ & 86.77 & $48.78 \mathrm{a}-\mathrm{c}$ \\
\hline 11. & DMH-10 & $38.3 \mathrm{ab}$ & $159.67 \mathrm{~b}-\mathrm{g}$ & $89.41 \mathrm{~b}-\mathrm{g}$ & 86.88 & $50.27 a-c$ \\
\hline 12. & DMH-13 & $42.0 \mathrm{a}$ & $180.00 \mathrm{ab}$ & $100.80 \mathrm{ab}$ & 82.47 & $49.87 \mathrm{a}-\mathrm{c}$ \\
\hline 13. & NAH-1137 & $36.8 \mathrm{a}-\mathrm{c}$ & $167.33 b-e$ & $93.71 b-e$ & 87.39 & $51.22 \mathrm{ab}$ \\
\hline 14. & NAH-2049 & $34.9 a-c$ & $163.33 b-f$ & $91.47 b-f$ & 87.93 & $50.51 \mathrm{a}-\mathrm{c}$ \\
\hline 15. & МАH-957 & $35.6 a-c$ & $171.33 \mathrm{a}-\mathrm{d}$ & $95.95 a-d$ & 87.48 & $49.89 a-c$ \\
\hline 16. & МАH-974 & $33.5 a-d$ & $154.67 \mathrm{c}-\mathrm{h}$ & $86.61 \mathrm{c}-\mathrm{h}$ & 88.07 & $53.79 \mathrm{a}$ \\
\hline 17. & DMH-04 & $31.3 b-d$ & 102.331 & 57.311 & 86.27 & $42.16 c$ \\
\hline 18. & DMH-06 & $29.2 \mathrm{~cd}$ & $125.33 \mathrm{j}-1$ & $70.19 \mathrm{j}-1$ & 91.70 & $48.97 \mathrm{a}-\mathrm{c}$ \\
\hline 19. & DMH-08 & $36.0 \mathrm{a}-\mathrm{c}$ & $112.67 \mathrm{kl}$ & $63.09 \mathrm{kl}$ & 85.24 & $44.38 \mathrm{bc}$ \\
\hline \multirow[t]{4}{*}{20.} & DMH-17 & $40.8 \mathrm{a}$ & $120.00 \mathrm{j}-1$ & $67.20 \mathrm{j}-1$ & 89.76 & $48.84 a-c$ \\
\hline & Mean & 36.54 & 147.58 & 82.65 & 86.65 & 49.13 \\
\hline & S.Em+ & 2.5 & 5.934 & 3.32 & 2.66 & 1.75 \\
\hline & $\operatorname{LSD}(5 \%)$ & 7.1 & 16.988 & 9.51 & NS & 5.01 \\
\hline
\end{tabular}

Note: DMRT-values in the column followed by the same letter do not differ significantly

DAS - Days after sowing 
Total soluble sugar content in corn is significantly varied with genotypes. Soluble sugar is main osmolytes in maize plants and they are used for osmotic adjustment. Increase in soluble sugar content in plant cells have been observed among the hybrids. The data on total soluble sugar content was presented in the (Table 2). The maximum total soluble sugar (2.92 $\mathrm{mg} \mathrm{g}^{-1}$ ) was recorded with DMH01 and which was statistically significant with other genotypes followed by DMH-13 (2.56 $\left.\mathrm{mg} \mathrm{g}^{-1}\right)$, GPMH-1101 (1.19 $\left.\mathrm{mg} \mathrm{g}^{-1}\right)$ and MAH-957 (1.10 $\left.\mathrm{mg} \mathrm{g}^{-1}\right)$. Whereas, lower total soluble sugar was recorded $\left(0.28 \mathrm{mg} \mathrm{g}^{-1}\right)$ with DMH-04 genotype. This is in agreement with the study by (Guanghua yin et al., 2012) and a parameter suitable to screening for drought tolerance in maize varieties.

Yield is the ultimate manifestation of morphological, physiological, biochemical processes and growth parameters and is considered to result from trapping and the conversion of solar energy efficiency. Improvement in yield can be realized in two ways i.e. by adopting the existing varieties to grow better in their environment by altering the relative proportion of different plant parts so as to increase the yield of economically important parts (Humphries, 1969).

Maize grain yield was affected by numerous other plant traits, Grain yield could be improved by selecting for the increased plant height, cob length, and 100 grain weight as reported by (Khan et al., 1999). Maize grain yield has high positive genetic association with ear length, kernel rows per ear, 1000kernel weight. Large variation in cob yield was noticed ranging from 57.31 to 107.71 quintals per hectare (Table 4). This indicates that the yield variability could be due to differential photosynthetic capacity or by differential dry matter production of respective genotypes. Ability of a genotype to produce reproductive apparatus is an important characteristic of a plant. Varietal difference for cob yield was due to variation in leaf carbon fixation (Jurgens et al., 1978) and could also be due to variation in grain number, kernel weight and cob length (Hall et al., 1981; Coasta et al., 1988).

Significant improvement in overall growth of the crop was due to increased photosynthetic efficiency which resulted in greater availability of photosynthates, metabolites and increased dry matter accumulation for growth and development of reproductive structures which has resulted in increased cob girth, cob length and cob weight.

DMH-01 registered significantly maximum cob weight (234.00g) over all the hybrids followed by DMH-13. Whereas, lower cob weight $(119.00 \mathrm{~g})$ was recorded with hybrid DMH-04. Cob girth was significantly higher $(17.71 \mathrm{~cm})$ in DMH-01 which was statistically on par with all other hybrids except DMH-04 (Table 3). While, DMH-04 recorded significantly lower cob girth (14.34 $\mathrm{cm}$ ) among all the hybrids. In the present study, maximum cob girth and cob length was recorded in DMH-01, which is in accordance with (Younas et al., 2002).

The maximum cob length $(18.12 \mathrm{~cm})$ was recorded with DMH-01 followed by $\mathrm{DMH}-$ 13, GPMH-1101, МАH-957, NAH-1137, NAH-2049 and DMH-10 which were statistically on par with each other and the least $(11.54 \mathrm{~cm})$ was recorded with $\mathrm{DMH}-$ 04.Number of seed rows per cob (17.47) was significantly higher in DMH-01followed by DMH-13, GPMH-1101, MAH-957, NAH1137 and NAH-2049 which were statistically on par with each other and the least number of seed rows per cob (12.67) was recorded with DMH-04. The number of seeds per cob was significantly higher (520.77) in hybrid DMH01 followed by GH-110204, MAH-957, NAH-2049, MAH-974 and NAH-1137 which 
were statistically on par with each other. While, DMH-17 recorded lower number of seeds (327.37) which was on par with DMH08, DMH-04, Arjun, GH-0727 and GPMH1111 hybrids.

Maximum test weight (43.3 g) was recorded with Arjun and which was statistically on par with each other in all maize hybrids and least (26.3g) was recorded with GH-110204. Significantly higher grain weight per plant (192.33g) was recorded with DMH-01 followed by DMH-13, GPMH-1101 and MAH-957, these hybrids recorded significantly superior over rest of hybrids. DMH-04 hybrid recorded lower yield per plant (102.33g). Yield per hectare was significantly higher (107.7 $\left.\mathrm{q} \mathrm{ha}^{-1}\right)$ in DMH-01 followed byDMH-13, GPMH-1101 and MAH957, which were statistically on par with each other and the least $\left(57.31 \mathrm{q} \mathrm{ha}^{-1}\right)$ was recorded with DMH-04.

Shelling percentage was significantly higher $(91.7 \%)$ in DMH-06 and which was recorded statistically on par with each other and the least shelling percentage $(82.21 \%)$ was recorded with DMH-01.Harvest index varied significantly with hybrids. The highest harvest index (53.79\%) in MAH-974, and which was on par with other hybrids except DMH-17 and DMH-08. Whereas, lowest harvest index was recorded with DMH-04 (42.16\%).

Based on the results it was concluded that genotype DMH -01 recorded higher plant height, number of leaves, leaf area and TDM and recorded higher SPAD value, Chlorophyll content, photosynthetic rate starch and total sugar and also yield components like cob weight its length, number of seed rows and kernels per cob, shelling percentage, harvest index, yield and yield components compared to other genotypes.

\section{References}

Balasubramanian, V., Morales, A., Cruz, R. T. and Abdulrahman, S.,, On farm adaptation of knowledge-intensive nitrogen management technology for rice systems. Nutr. Cycle Agroecol., 53: 93-101 (1999).

Banziger, M. and Araus, J. L., Advances in molecular breeding toward drought and salt tolerant crops. Wat. Manage, 80:212- 224 (2007).

Coasta,J.O., Ferraria,L.G.R. and Souza, F.D., Yield of maize under different levels of water stress. Pesquisa Agropecuria Brasileiria, 23: 1255-1261(1988).

Dass, S., Arora, P., Kumari, M. and Dharma, P. Morphological traits determining drought tolerance in maize (Zea mays L.). Indian J. Agric. Res., 35 (3): 59-63 (2008).

Guang-hua Yin, Ye-jieShen, Na Tong, Jian $\mathrm{Gu}$, Liang Hao and ZuoXin Liu, J. Food Agric. Environ., 10 (1): 853-858( 2012).

Hader, Association of various physiomorphological characters in maize (Zea mays L.). M. Sc. (Hons.) Agri. Thesis, Univ. Agri., Faisalabad, Pakistan., (2006).

Hall, A. J., Lemcoff, J.H., Tra pani, N. Water stress before and during flowering in maize and its effects on yield, its components, and their determinants. Maydica 26:19-38(1981).

Hiscox and Israeltam, A method for extraction of chlorophyll from leaf tissue without maceration. Canadian J. Bot., 57:1332 1334(1979).

Jurgens, S.K., Johnson, R.R. and Boyer, J.S., Dry matter production and translocation in maize subjected to drought during grain fill. Agron. J., 70: 678-688(1978).

Khan, M., Akbar, S., Ahmad, K. and Baloch, M. S., Evaluation of corn hybridsfor grain yield in D. I. Khan. Pak. J. Biol. Sci., 2 (2): 413-414 (1999). 
Manish Kumar, Growth, yield and water use efficiency of different winter maize (Zea mays L.) varieties as influenced by nitrogen and irrigation scheduling. M. Sc. Thesis, Indira Gandhi Agricultural University, Raipur (1998).

Modarres, A. M., Hamilton, R. I., Dwyer, L. M., Stewart, D. W., Mather, D. E., Dijak, M. and Smith, D. L., Leafy reducedstature maize for short-season environments: Morphological aspects of inbred lines. Euphytica, 96 (2): 301-309 (1997).

Pellizzaro, G., Venture, A., Arca, B. and Crlu, A non-destructive method to determine leaf chlorophyll content in grain sorghum. Agricul. Mediterranea, 128 (4): 330-338(1998).

Peng, S., Garcia, F. V., Laza, R. C., Sanico,
A. L., Visperas, R. M. and Cassman, K. G., Increased N-use efficiency using a chlorophyll meter on high yielding irrigated rice. Field Crops Res., 47: 243 -252 (1996).

Saleem, A., Saleem, U. and Subhani, G. M., Correlation and path coefficient analysis in maize (Zea mays L.). J. Agric. Res., 45 (3): 450-459 (2007).

Younas, M., Rehman, H. and Hayder, G., Magnitude of variability for yield andyield associated traits in maize hybrids. Asian J. Plant Sci. 1 (6): 694696(2002).

Yunfeng, P., Li, C. and Felix, B. F., Diurnal dynamics of maize leaf photosynthesis and carbohydrate concentrations in response to differential $\mathrm{N}$ availability. Environ. Exptl. Bot., 99: 18-27(2014).

\section{How to cite this article:}

Pawar, K. N. and Goudar, A. L. 2020. Evaluation of Different Maize Hybrids for Morphophysiological, Biochemical and Yield and Yield Components. Int.J.Curr.Microbiol.App.Sci. 9(12): 2472-2480. doi: https://doi.org/10.20546/ijcmas.2020.912.292 